\title{
Tumormarker (CEA, CA 15-3)- und Steroidhormonrezeptor-Bestimmungen im Zytosol von Mammakarzinomen
}

\author{
Tumor Markers (CEA, CA 15-3) and Steroid Receptors of Carcinoma of the Breast
}

Sabine Eisenhut, E. Kreuzfelder, N. Scheiermann, O. Thraenhart

Institut für Medizinische Virologie und Immunologie (Komm. Direktor: PD Dr. O. Thraenhart)

Klinikum der Universität - Gesamthochschule - Essen

\begin{abstract}
Zusammenfassung:
In Zytosolen von 168 Patientinnen mit Mammakarzinomen wurden carcinoembryonales Antigen (CEA), CA 15-3 und Steroidhormonrezeptoren bestimmt. Es zeigte sich, daß die CEA-Konzentrationen im Zytosol weder mit den Konzentrationen von CA 15-3 und Steroidhormonrezeptoren noch mit histologischen Kenngrößen, Alter oder Menopausen-Status korrelierte. Neben dem bekannten prognostischen Wert der Estrogenrezeptoren ergaben sich Hinweise für eine prognostische Bedeutung des CA 15-3.
\end{abstract}

Schlüsselwörter:

CEA - CA 15-3-Mammakarzinom - Steroidhormonrezeptoren - Zytosol

Summary:

Carcinoembryonic antigen (CEA), CA 15-3 and steroid receptors were estimated in tumors from 168 patients with primary breast cancer. After evaluation of tumor marker measurement in cytosols it was shown that CEA concentrations were not related nor to the CA 15-3 concentrations or steroid receptors amounts neither to the TNM system, grade of tumor differentiation or age and menopausal status. Aside from the known prognostic value of estrogen receptor determination, measurement of CA 15-3 in cytosols may have a prognostic significance.

Keywords:

CEA - CA 15-3 - breast cancer - cytosol - steroid receptors

\section{Einleitung}

Carcinoembryonales Antigen (CEA) kann als Prototyp eines Tumormarkers betrachtet werden, da es sowohl zur Karzinomdiagnostik wie auch für die Therapieüberwachung eingesetzt werden kann (1). Darüber hinaus erlaubt es auch prognostische Aussagen (1).

Erhöhte CEA-Konzentrationen im Serum werden besonders bei kolorektalen Karzinomen beobachtet. Dagegen werden im Serum von Patientinnen mit Mammakarzinomen im Durchschnitt nur 30\% erhöhte Werte gefunden (2).

Im Gegensatz zur Bestimmung im Serum liefert die CEABestimmung im Tumorgewebe, $\cdot d$. h. im Zytosol der aufgearbeiteten Zellen, wesentlich öfter erhöhte Werte $(3,4$, 5). Obwohl dies weiterführende Untersuchungen etwa zur Definition von Risikogruppen mittels der CEA-Bestimmung im Zytosol nahelegt, ist die Zahl derartiger Untersuchungen eher klein geblieben. Darüber hinaus wurden unterschiedliche Ergebnisse erhalten. In einer Studie konnten die Untersucher bei 62 Mammakarzinomen keine Beziehung zwischen Stadium oder histologischen Klassifizierungsmerkmalen und der zytosolischen CEAKonzentration erkennen (3), während in einer zweiten Untersuchung von 259 primären Karzinomen signifikant erhöhte CEA-Werte im Zytosol fortgeschrittener Tumoren gefunden wurden (6). Es wurde auch eine Korrelation der Konzentrationen von CEA und Estrogenrezeptoren beschrieben (4); dies konnte in einer zweiten Untersuchung nicht bestätigt werden (5). Obwohl CA 15-3 die Primärdiagnose und die Verlaufskontrolle des Mammakarzinoms verbessert $(7,2)$, liegen keine Untersuchungen über seine Konzentration im Tumor-Zytosol vor.

Wir bestimmten deshalb CA 15-3 und CEA im Zytosol von Mammakarzinomen und verglichen diese Werte mit Daten von solchen Kenngrößen, die prognostischen Wert beim Mammakarzinom besitzen.

\section{Material und Methoden}

Im Rahmen der Rezeptordiagnostik beim Mammakarzinom wurden ultrazentrifugierte Gewebsextrakte, Zytosole, von 168 Patientinnen mit Mammakarzinomen (Altersmedian: 66 Jahre, Bereich 25 bis 87 Jahre) gewonnen. Die einsendenden Kliniken stellten Informationen bezüglich der histologischen Klassifizierung der Tumoren und der klinischen Einordnung der Patientinnen zur Verfügung.

Nach dem histologischen Nachweis eines Mammakarzinoms wurde ein Teil des Tumors bis zur Estrogenrezeptor (ER)-. und Progesteronrezeptor (PgR)-Bestimmung nach den EORTEC-Vorschriften (8) in flüssigem Stickstoff gelagert.

Die CEA-Konzentrationen wurden parallel mit einem Enzymimmunoassay (EIA) der Firma Hoffmann-La Roche, Grenzach-Wyhlen, und mit einem Lumineszenz-verstärkten (Lv) EIA (Amerlite) der Firma Amersham Buchler, 
Braunschweig, bestimmt. Beide Testsysteme verwenden einen monoklonalen anti-CEA Antikörper. Die CA 15-3Konzentrationen wurden mit Hilfe eines Radioimmunoassays der Firma Isotopendiagnostik CIS, Dreieich, ermittelt. Zur Validierung der CEA- und CA 15-3-Bestimmungen wurden in jeweils einem Zytosol die beiden Tumormarker mit und ohne Zusatz eines entsprechenden Stan. dards bestimmt. In einem weiteren Zytosol wurden erhöhte CEA- und CA 15-3-Werte nach Verdünnung mit dem 0-Standard (CEA) bzw. Verdünnungsmedium überprüft.

Die Proteinkonzentration wurde entsprechend (9) bestimmt.

Unterschiede zwischen den verschiedenen PatientinnenGruppen wurden mit dem Kruskal-Wallis- (mehr als zwei Gruppen) bzw. dem Wilcoxon-Test geprüft. Korrelationen wurden mit Hilfe des Spearmanschen Korrelationskoeffizienten beurteilt, wobei Korrelationskoeffizienten von $r>0,5$ als aussagekräftig erachtet wurden. Die Signifikanzschranke wurde bei zweiseitigen Testbedingungen auf $p<0,05$ festgelegt. Die Berechnungen wurden mit einem IBM 4331-Rechner mit dem Statistical Analysis System (SAS) unter VM/SP durchgeführt.

\section{Ergebnisse}

Ein "Matrixeffekt" bei der Zytosolmessung ist wahrscheinlich, da sowohl bei Zusatz von CEA als auch CA 153 im Zytosol nur etwa $70 \%$ dieser Tumormarker wiedergefunden wurden (Tab. 1). Über dem Meßbereich liegende Werte konnten nach Verdünnung gemessen werden (Tab. 2). Die mit den beiden CEA-Testsystemen erhaltenen 164 Wertepaare korrelieren mit einem Korrelationskoeffizienten von $r=0,94$ signifikant $(p<0,0001)$. Die mit dem (LV)EIA gemessenen CEA-Konzentrationen (165 Werte, Mittelwert $93 \mu \mathrm{g} / \mathrm{l}$, Standardabweichung $266 \mu \mathrm{g} / \mathrm{l})$ sind signifikant ( $p<0,0001$ ) niedriger als die mit dem zweiten Testsystem erhaltenen (167 Werte, Mittelwert 138 $\mu \mathrm{g} / \mathrm{l}$, Standardabweichung $389 \mu \mathrm{g} / \mathrm{l})$. Die Präzision beider CEA-Meßsysteme ist vergleichbar, da der Variationskoeffizient für die o. g. Meßwerte beim (LV)EIA $286 \%$ und beim EIA $282 \%$ beträgt.

Unterschiede in den CEA-Konzentrationen wurden bei nach dem TNM-Klassifizierungssystem, Differenzierungsgrad der Tumoren, Alter und dem Menopausen-Status unterschiedenen Patientinnen-Gruppen mit beiden Testen nicht beobachtet. Die Patientinnen-Gruppe mit lokalisierten Mammakarzinomen (T1-4NOMO) zeigte dagegen signifikant höhere CA 15-3-Konzentrationen (bezogen auf $\mathrm{mg}$ Zytosolprotein) als die Gruppe mit metastasierten Karzinomen (T1-4N1-3M1) (Tab. 3); allerdings war die Proteinkonzentration der Zytosole beider Gruppen si-

Tab. 1: "Präzision in der Serie" und Wiederfindung bei 10fachBestimmung von CEA und CA 15-3 in Mammakarzinomzytosolen.

\begin{tabular}{|c|c|c|c|c|c|c|c|}
\hline \multirow[t]{3}{*}{ Meßgröße } & \multicolumn{7}{|c|}{ Cytosolkonzentration } \\
\hline & \multicolumn{3}{|c|}{ Ohne Standardzusatz } & \multicolumn{3}{|c|}{ Mit Standardzusatz } & \multirow[t]{2}{*}{ Sollwert } \\
\hline & $\bar{x}$ & SD & VK (\%) & $\overline{\mathbf{x}}$ & SD & VK (\%) & \\
\hline $\begin{array}{l}\text { CEA } \\
(\mu \mathrm{g} / \mathrm{l})\end{array}$ & 0 & - & - & 7,2 & 1,8 & 25 & 10 \\
\hline $\begin{array}{l}\text { CA 15-3 } \\
\text { (kU/I) }\end{array}$ & 91 & 9 & 10 & 45 & 5 & 11 & 66 \\
\hline
\end{tabular}

SD: Standardabweichung. VK: Variationskoeffizient. $\bar{x}$ : Mittelwert. gnifikant verschieden (Tab. 3). Die CA 15-3-Konzentrationen korrelierten weder mit dem Alter, dem MenopausenStatus noch mit den histologischen Klassifizierungsmerkmalen. Auch die CA 15-3- und CEA-Konzentrationen korrelierten nicht.

Signifikant niedrigere ER-Konzentrationen wurden bei $\mathrm{Pa}$ tientinnen mit axillärem Lymphknotenbefall gemessen (Tab. 4). Der Korrelationskoeffizient für ER- und PgRKonzentrationen betrug für 162 Parallelbestimmungen $r=0,52(p<0,001)$. Darüber hinaus zeigten wenig differenzierte Tumoren (G3) bei ähnlichen Proteinkonzentrationen signifikant niedrigere ER-Konzentrationen als G1und G2-Mammakarzinome (Tab. 5). Ferner waren die ERKonzentrationen bei 112 Patientinnen im Alter von 60 bis 89 Jahren signifikant $(p<0,005)$ höher als bei den 5420 bis 59jährigen. Zudem fanden sich bei 85 Patientinnen 10 bis 14 Jahre nach Menopause signifikant $(p<0,05)$ höhere ER-Konzentrationen im Zytosol als bei 27 Patientinnen bis zu neun Jahren nach Menopause.

\section{Diskussion}

Die Bestimmung der "Präzision in der Serie” weist sowohl bei CEA wie auch bei CA 15-3 auf einen "Matrixeffekt" des Zytosols hin, da nach Zugabe von Standardmarterial die theoretischen Werte nicht erhalten wurden. Ein "Matrixeffekt" könnte auch die im Vergleich zum Serum $(10,11)$ höheren Variationskoeffizienten der CEA- und CA 15-3-Zytosolmessung erklären.

Wichtige prognostische Faktoren für die Verlaufsbeurteilung von Mammakarzinomen sind der axilläre Lymphknoten-Status und - in geringerem Maße - histologische Klassifizierungsmerkmale sowie die ER-Zytosolkonzentration (12). Keine dieser Prognostischen Kenngrößen korrelierte mit Tumormarker-Konzentrationen in den Zytosolen. Zur Frage der Korrelation der Zytosolkonzentrationen von CEA und ER wurden zwei Arbeiten mit widersprüchlichen Ergebnissen publiziert $(4,5)$.

Die CA 15-3-Zytosolkonzentrationen von Tumoren, die bereits in die axillären Lymphknoten metastasierter („Lymphknoten-positiv"), waren signifikant niedriger als die der "Lymphknoten-negativen". Niedrigere CA 15-3Werte bei "Lymphknoten-positiven" Tumoren könnten mit einem vermehrten Übertritt von CA 15-3 aus dem Gewebe in die Zirkulation erklärt werden (7). Da der Medianwert der CA 15-3-Konzentrationen „Lymphknoten-positiver" Tumoren erniedrigt ist, das Ausmaß der Erniedrigung aber nicht der Verminderung der Proteinkonzentration entspricht, scheint im Zytosol dem CA 15-3 in Verbindung mit der Proteinkonzentration eine prognostische Bedeutung zuzukommen.

Tab. 2: Verdünnungsreihe mit Einfach-Bestimmung von CEA und CA 15-3 von einem Mammakarzinomzytosol.

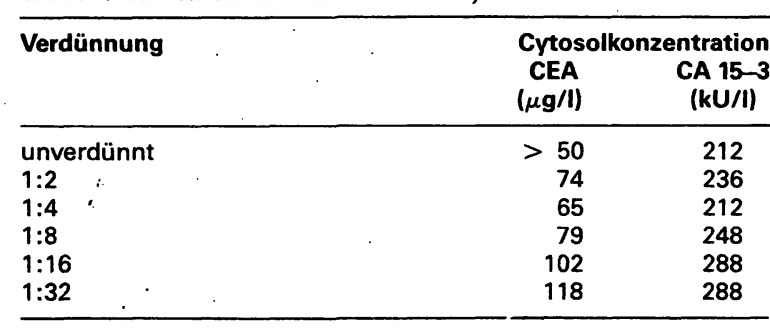

Zur Verdünnung wurde beim CEA der 0-Standard und beim CA 15-3 ein gesondertes Verdünnungsmedium benutzt. 
Tab. 3: CEA und CA 15-3 im Zytosol von Patientinnen mit lokalisierten (T1-4 NO MO) und metastasierten (T1-4 N1-3 M1) Mammakarzinomen.

\begin{tabular}{|c|c|c|c|c|c|}
\hline \multicolumn{2}{|c|}{ Tumormarker } & \multicolumn{3}{|c|}{ Mammakarzinom-Patientinnen } & \multirow{2}{*}{$\begin{array}{l}\text { p-Wert } \\
\text { (T1-4 NO MO vs. } \\
\text { T1-4 N1-3 M1) }\end{array}$} \\
\hline & & Alle & TI-4 NOMO & T1-4 N1-3M1. & \\
\hline $\begin{array}{l}\text { CEA EIA } \\
(\mu \mathrm{g} \cap)\end{array}$ & $\begin{array}{l}\text { M Median } \\
\text { Bereich }\end{array}$ & $\begin{array}{l}135 \\
22,2 \\
0,5-1687\end{array}$ & $\begin{array}{l}71 \\
22,5 \\
2,1-1687\end{array}$ & $\begin{array}{l}64 \\
21,5 \\
0,5-1160\end{array}$ & NS \\
\hline $\begin{array}{l}\text { ( } \mu g / g \\
\text { Protein) }\end{array}$ & $\begin{array}{l}n \\
\text { Median } \\
\text { Bereich }\end{array}$ & $\begin{array}{l}135 \\
11,8 \\
0,3-530\end{array}$ & $\begin{array}{l}71 \\
11,6 \\
1,4-530\end{array}$ & $\begin{array}{l}64 \\
13,5 \\
0,3-476,3\end{array}$ & NS \\
\hline $\begin{array}{l}\text { CEA LvEIA } \\
(\mu g /)\end{array}$ & $\begin{array}{l}n \\
\text { Median } \\
\text { Bereich }\end{array}$ & $\begin{array}{l}135 \\
18,4 \\
0-2013\end{array}$ & $\begin{array}{l}71 \\
19,2 \\
0-1759\end{array}$ & $\begin{array}{l}64 \\
14,1 \\
0-2013\end{array}$ & NS \\
\hline $\begin{array}{l}\text { ( } \mu \mathrm{g} / \mathrm{g} \\
\text { Protein) }\end{array}$ & $\begin{array}{l}\mathrm{n} \\
\text { Median } \\
\text { Bereich }\end{array}$ & $\begin{array}{l}135 \\
9,9 . \\
0-606,6\end{array}$ & $\begin{array}{l}71 \\
10,4 \\
0-606,6\end{array}$ & $\begin{array}{l}64 \\
9,0 \\
0-544,1\end{array}$ & NS \\
\hline $\begin{array}{l}\text { CA 15-3 } \\
\text { (kUN) }\end{array}$ & $\begin{array}{l}n \\
\text { Median } \\
\text { Bereich }\end{array}$ & $\begin{array}{l}138 \\
102 \\
0-1848\end{array}$ & $\begin{array}{c}72 \\
110 \\
0-1848\end{array}$ & $\begin{array}{l}66 \\
88 \\
0-1759\end{array}$ & NS \\
\hline $\begin{array}{l}\text { (kU/g } \\
\text { Protein) }\end{array}$ & $\begin{array}{l}n \\
\text { Median } \\
\text { Bereich }\end{array}$ & $\begin{array}{l}138 \\
50 \\
0-1175\end{array}$ & $\begin{array}{l}72 \\
68 \\
0-1175\end{array}$ & $\begin{array}{l}66 \\
38 \\
0-1043\end{array}$ & 0,0275 \\
\hline $\begin{array}{l}\text { Protein- } \\
\text { gehalt } \\
\text { (g/) }\end{array}$ & $\begin{array}{l}n \\
\text { Median } \\
\text { Bereich }\end{array}$ & $\begin{array}{l}138 \\
2,3 \\
0,3-6,7\end{array}$ & $\begin{array}{l}72 \\
2,0 \\
0,3-6,7\end{array}$ & $\begin{array}{l}66 \\
2,5 \\
0,7-6\end{array}$ & 0,0198 \\
\hline
\end{tabular}

EIA : Enzymimmunoassay. Lv: Lumineszenz-verstärkt. $n$ : Patientinnenzahl.
Tab. 4: Steroidhormonrezeptoren im Zytosol von Patientinnen mit lokalisierten (T1-4 NO MO) und metastasierten (T1-4 N1-3 M1) Mammakarzinomen.

\begin{tabular}{|c|c|c|c|c|}
\hline \multirow{2}{*}{$\begin{array}{l}\text { Steroidhormon- } \\
\text { rezeptor }\end{array}$} & \multicolumn{3}{|c|}{ Mammakarzinom-Patientinnen } & \multirow{2}{*}{$\begin{array}{l}\text { p-Wert (T1-4 } \\
\text { NO MO versus } \\
\text { T1-4 N1-3 M1 }\end{array}$} \\
\hline & Alle & TI-4 NO MO & $\mathrm{T} 1-4 \mathrm{N1}-3 \mathrm{M1}$ & \\
\hline \multicolumn{5}{|l|}{ Estrogen } \\
\hline Anzahl & 136 & 70 & 66 & \\
\hline $\begin{array}{l}\text { Median } \\
\text { (fmol/mg Protein) }\end{array}$ & 34 & 42 & 17 & 0,0128 \\
\hline $\begin{array}{l}\text { Bereich } \\
\text { (fmol/mg Protein) }\end{array}$ & $\begin{array}{r}0- \\
650\end{array}$ & $\begin{array}{r}0- \\
650\end{array}$ & $\begin{array}{l}0- \\
333\end{array}$ & \\
\hline \multicolumn{5}{|l|}{ Progesteron } \\
\hline Anzahl & 136 & 70 & 66 & \\
\hline $\begin{array}{l}\text { Median } \\
\text { (fmol/mg Protein) }\end{array}$ & 77 & 93 & 53 & 0,0445 \\
\hline $\begin{array}{l}\text { Bereich } \\
\text { (fmol/mg Protein) }\end{array}$ & $\begin{array}{l}0- \\
947\end{array}$ & $\begin{array}{c}0- \\
\cdot \quad 947\end{array}$ & $\begin{array}{c}0- \\
662\end{array}$ & \\
\hline
\end{tabular}

Tab. 5: Estrogenrezeptoren im Zytosol von Patientinnen mit Tumoren unterschiedlicher Differenzierung.

\begin{tabular}{lrrrrr}
\hline Estrogenrezeptor & Alle & \multicolumn{3}{c}{ Klassifizierung } & p-Wert \\
& & G1 & G2 & G3 & (G3 versus G2) \\
\hline Anzahl & 140 & 18 & 76 & 46 & \\
Median & 34 & 37 & 48 & 12 & 0,0136 \\
(fmol/mg Protein) & $0-$ & $0-$ & $0-$ & $0-$ & \\
$\begin{array}{l}\text { Bereich } \\
\text { (fmol/mg protein) }\end{array}$ & 740 & 589 & 570 & 74 & \\
\hline
\end{tabular}

Die gleiche Argumentation gilt auch für die prognostische Bedeutung der Steroidhormonrezeptor-Bestimmung beim Mammakarzinom. Obwohl ER- und PgR-Konzentrationen in unseren Untersuchungen ebenso wie bei (13) korrelieren, ist die Bedeutung beider Rezeptoren unterschiedlich: wenig differenzierte G3-Mammatumore zeigen ähnliche PgR-Werte, aber signifikant niedrigere ERWerte als G1- und G2-Tumoren. In Über.einstimmung mit publizierten Ergebnissen $(14,15,16)$ sind nach unseren Ergebnissen auch Alter und Menopausen-Status mit der. ER-Konzentration zu ass̄ożiieren. Offenbar wird die ERKonzentration durch Größen beeinflußt, die nicht unmittelbar mit dem Tumor in Zusammenhang zu bringen sind.
Anschrift des Verfassers:

PD Dr. rer. nat. Ernst Kreuzfelder

Sabine Eisenhut

PD Dr. med. Norbert Scheiermann

PD Dr. med. vet. Olaf Thraenhart

Institut für Medizinische Virologie und Immunologie

Universitätsklinikum - Gesamthochschule - Essen

Hufelandstraße 55

4300 Essen 1
Schriftum:

1. SCULIER, J. P., BODY, J. J., JACOBOWITZ, D., FRUHLING, J.: Value of CEA determination in biological fluids and tissues. Eur. J. Cancer Clin. Oncol. 23, 1091-1093 (1987). 2. KREIENBERG, R.: Allgemeine und spezifische Laborparameter im Rahmen der Tumornachsorge bei gynäkologischen Malignomen und bei Mammakarzinomen. Gynäkologe 22, 55-62 (1989).

3. DUFFY, M. O'CONNELL, M. O'SULLIVAN, F., McKENNA, B., ALLEN, M. A., MCDONNELL, L.: CEA-like material in cytosols from human breast carcinomas. Cancer MCDONNELL, L.: $121-123$ (1983).

4. GION, M., MIONE, R., DITTADI, R., FASAN, S., PALLINI, A., BRUSCAGNIN, G.: Carcinoembryonic antigen, ferritin, and tissue polypeptide antigen in serum and tissue. Cancer 57, 917-922 (1986).

5. MENENDEZ-BOTET, C. J., NISSELBAUM, J. S., FLEISHER, M., ROSEN, P. P., FRACCHIA A ROBBINS, G. URBAN, J. A., SCHWARTZ, M. K.: Correlation between estrogen reptor protein and carcinoembryonic antigen in normal and carcinomatous hugen receptor protein and carcinoem 1366-1371 (1976).

man breast tissue. Clin. Chem. 22, 1366-1371 6. SCHWARTZ, M. R., RANDOLPH, R. L., PANKO, W. B.: Carcinoembryonic antigen and
steroid receptors in the cytosol of carcinoma of the breast. Cancer 55, 2464-2471

(1985).

7. RODECK, U., FUNK, R., KREUZFELDER, E:, SCHEIERMANN, N.: Tumormarker (CA 15-3, CEA)-gestützte präoperative Diagnostik und Verlaufsbeobachtungen von Mammakarzinomen. Lab.med. 11, 224-226 (1987).

8. E. O. R. T. C. Breast Co-operative Group: Revision of the standards for the assessment of hormone receptors in human breast cancer; report of the second E. O. R. T. C. mork workshop, held on 16-17 marc

Cancer 16, 1513-10 9. LOWRY, O. H., ROSENBROUGH, N. J., FARR, A. L., RANDALL, R. J.: Pro
ment with the folin phenol reagent. J. Biol. Chem. 193, 265-275 (1951).

10. KUWERT, E., KREUZFELDER, E., RODECK, U., HENBEN, U. B.: Carcinoembryonales Antigen (CEA): Diagnostische Sensitivităt und Methodik verschiedener enzym- und radioimmunologischer Bestimmungen beim Mammakarzinom. TumorDiagnostik \& Therapie 6, 116-119 (1985).

11. BARAK, M., STEINER, M., FINKEL, B., ABRAHAMSON, J., ANTAL, S., GRUENER, N.: CA-15.3, TPA and MCA as markers for breast cancer. Europ. J. Cancer 26, 577-580 (1990).

12. CONTESSO G SACCANIJOTT! G, BONADONNA, G.: Tumor grade as a progno12. CONTES , G., SACCast cancer. Eur. J. Cancer Clin. Oncol. 25, 403-409 (1989). stic factor in primary breast cancer. Eur. J. Cancer Clin. Oncol. 25, 403-409 (1989). 13. ALEXIEVA-FIGUSCH, J., VAN PUTTEN, W. L. J., BLANKENSTEIN, M. A., BLONKVAN DER WIJST, J., KLIJN, J. G. M.: The prognostic value and relationships of patient
characteristics, estrogen and progestin receptors, and site of relapse in primary breast cancer. Cancer 61, 758-768 (1988).

14. LESSER, M. L., ROSEN, P. P., SENIE, R. T., DUTHIE, K., MENENDEZ-BOTET, C. SCHWARTZ, M. K.: Estrogen and progesterone receptors in breast carcinoma: correlations with epidemiology and pathology. Cancer 48, 299-309 (1981).

15. PARL, F. F, WAGNER, R. K. The histopathological evaluation of human breast can15. PARL, F. F., WAGNen,

cers in correlation with estrogen receptor values. Cancer $46,362-367$ (1980). . Study Surgery and Pathology Associates. Prognostic value of estrogen receptor determinations in patients with breast cancer. Cancer Res. 38, 4296-4298, 147-148 (1978). 